\title{
Obesidade infantil e suas consequências: uma revisão da literatura
}

\section{Children obesity and its consequences: a literature review}

\author{
Gisele Bailich Capistrano ${ }^{1}$, Mariana Marcolino Costa $^{2}$, André Everton de Freitas ${ }^{1,3}$, Paulo
} Roberto Santos Lopes ${ }^{1,3}$, Ana Inês Gonzáles ${ }^{1,3}$, Anelise Sonza ${ }^{3}$, Joel Alves Lamounier ${ }^{2}$.

\begin{abstract}
RESUMO
Dentre muitas patologias, atualmente a obesidade apresenta uma significativa prevalência no Brasil e no mundo. Considerada uma doença crônica que resulta no aumento de gordura, proveniente de um desequilíbrio no balanço energético, que vem acometendo crianças e adolescentes causando graves consequências cardiovasculares, metabólicas e psicossociais. Este estudo tem objetivo de promover uma reflexão teórica sobre a obesidade infantil e identificar os aspectos gerais associados a esta patologia, destacando aspectos epidemiológicos, etiológicos, consequências e tratamento, considerando a importância da atividade física (AF). Durante a revisão da literatura, construindo os objetivos deste estudo, alcançamos alguns pilares na compreensão da doença, em que se verificou a importância de uma equipe multidisciplinar, promovendo a saúde e prevenir a doença, o que implica a alteração de hábitos de vida, como também os fatores culturais, sociais e ambientais.
\end{abstract}

Palavras-chave: Obesidade infantil; atividade física; qualidade de vida.

\begin{abstract}
Among many pathologies, obesity currently has a significant prevalence in Brazil and in the world. It's considered a chronic disease that results in increased fat, resulting from an imbalance in energy balance, which has been affecting children and adolescents and it may cause serious cardiovascular, metabolic and psychosocial consequences. This study aims to promote a theoretical reflection on children obesity and identify the general aspects associated with this pathology, highlighting epidemiological and etiological aspects, consequences and treatment, it also considers the importance of physical activity (PA). During the literature review, building the objectives of this study, we reached some pillars in this disease understanding, in which it was verified the importance of a multidisciplinary team, to promote health and prevent this disease, which implies the change of life habits, such as cultural, social and environmental factors.
\end{abstract}

Keywords: Childhood obesity; physical activity; quality of life.

\footnotetext{
${ }^{1}$ UNIDAVI: Centro Universitário para o Desenvolvimento do Alto Vale do Itajaí. gisele.capistrano@unidavi.edu.br

2 UFSJ: Universidade Federal de São João Del - Rei

${ }^{3}$ UDESC: Universidade do Estado de Santa Catarina - Centro de Ciências da Saúde e do Esporte (CEFID)
} 


\section{INTRODUÇÃO}

O sobrepeso e a obesidade são considerados problemas de saúde no mundo, atingindo crianças, adolescentes, adultos e idosos de ambos os sexos. No panorama mundial e brasileiro, o excesso de peso vem enfrentando um aumento significativo que são responsáveis por complicações consideráveis (MENEZES e VASCONCELOS, 2021) e demonstrando um cenário desafiador à saúde, atualmente tornando-se problema na saúde pública mais prevalente (KUMAR e KAUFMANN, 2018).

Nos últimos anos, no Brasil, observou-se prevalência nos indicadores do excesso de peso na população infantil, estimando-se que 9,4\% das meninas e $12,4 \%$ dos meninos, foram considerados obesos em 2020 (SBC, 2020), sendo que o número de crianças obesas de 5 a 9 anos cresceu mais de 300\% entre os anos de 1989 e 2009 (CRESCENTE et al., 2021).

Algumas das consequências da obesidade estão relacionadas a um maior risco para doenças crônicas não transmissíveis (DCNT). Além disso, segundo a Sociedade Brasileira de Pediatria (SBP), na infância, o excesso de peso está diretamente ligado às concentrações elevadas de insulina plasmática, perfil lipídico alterado e hipertensão arterial (GURNANI et al., 2015). Essa incidência pode ter impacto na expectativa de vida, implicando no crescimento e desenvolvimento musculoesquelético (SBP, 2012).

A atividade física (AF) aliada a uma boa alimentação, desempenham um papel fundamental no estilo de vida e na composição corporal, podendo regular o perfil lipídico e metabólico (PAES, MARINS e ANDREAZZI, 2015). Além disso, os benefícios da AF não ficam restritos apenas à questão física, mas também aos aspectos psicológicos, cognitivos e socioculturais (OLIVEIRA et al., 2011).

Neste sentido, estudos demonstram que crianças que manifestam sobrepeso, apresentam maior risco de se tornarem adultos obesos, deste modo, a prevalência de excesso de peso em crianças e adolescentes pode levar a diminuição na qualidade de vida elevando o custo em cuidados com saúde (JARDIM e SOUZA, 2017).

\section{METODOLOGIA}

Esta pesquisa trata-se de uma revisão de literatura de caráter qualitativo, ou seja, não requer uso de técnicas estatísticas e não pode ser traduzido em números. $O$ levantamento bibliográfico foi conduzido nas bases de dados PubMed, Scielo e Google Acadêmico, onde os estudos variam entre os anos de 2003 à 2021. As palavras usadas 
para a pesquisa foram: "obesidade", "sobrepeso", "atividade física", "escolares", “obesity", "overweight", "physical activity", "school children".

Foram selecionados apenas os artigos que tinham interesse para o objetivo proposto, ou seja, promover uma reflexão teórica sobre a obesidade infantil e identificar os aspectos gerais associados a esta patologia, destacando aspectos epidemiológicos, etiológicos, consequências e tratamento, considerando a importância da AF.

\section{RESULTADOS E DISCUSSÕES}

\section{Aspectos Epidemiológicos da Obesidade}

Segundo a Organização Mundial da Saúde (OMS), afirma que a obesidade é um dos mais graves problemas de saúde que temos que enfrentar. Em 2025, a estimativa é de que 2,3 bilhões de adultos ao redor do mundo estejam acima do peso, sendo 700 milhões de indivíduos com obesidade (ABESO, 2019).

No Brasil, de acordo com o Sistema de Vigilância de Fatores de Riscos e Proteção para a Doenças Crônicas por Inquérito Telefônico (VIGITEL), essa doença aumentou $72 \%$ nos últimos treze anos, saindo de 11,8\% em 2006 para 20,3\% da população em 2019 (VIGITEL, 2020).

Em adolescente, indica a prevalência de $15 \%$ a $19 \%$ no excesso de peso (CONDE, 2006. Apud SBP, 2019). Já em relação à obesidade infantil, o Ministério da Saúde e a Organização Panamericana da Saúde apontam que 12,9\% das crianças brasileiras entre 5 e 9 anos de idade têm obesidade, assim como 7\% dos adolescentes na faixa etária de 12 a 17 anos (ABESO, 2019).

Um estudo em escala global, liderado pelo Imperial College of Science, Technology and Medicine, de Londres, e pela OMS, avaliou 128,9 milhões de crianças de 1975 a 2016, constatando que a prevalência da obesidade padronizada por idade aumentou. Isso implica em um aumento de 0,7\% em 1975 para 5,6\% em 2016 de obesidade em meninas de 5 a 19 anos, e de $0,9 \%$ em 1975 para 7,8\% em 2016 de meninos obesos na mesma faixa etária (NCD-RISC et al., 2017).

Segundo dados da SBP, quatro em cada cinco crianças obesas permanecerão obesas quando adultas (SBP, 2012). A literatura também refere a idade próxima dos seis anos como um dos períodos mais críticos no desenvolvimento de mais acúmulo de gordura corporal e formação dos hábitos de vida (ABESO, 2016). 
Nessa perspectiva, é importante observar que o quadro epidemiológico nutricional deve ser apontado na prevenção primária para estratégias de saúde pública capazes de dar conta de um modelo de atenção para problemas relacionados à desnutrição e obesidade, abrangendo consequências e interfaces das políticas públicas dentro do processo de vida da população.

\section{Etiologia da Obesidade}

A obesidade etiologicamente é considerada multifatorial, pois têm interação entre fatores genéticos, metabólicos, nutricionais, psicossociais, ambientais e as mudanças no estilo de vida. (CAMPAGNOLO, et al., 2008. Apud SBP, 2019). Ela pode estar associada a síndromes genéticas ou alterações endócrinas metabólicas, caracterizado por um estado inflamatório crônico e acúmulo excessivo de gordura corporal (PEREIRA-LANCHA et al., 2012).

Segundo CARVALHO et al. (2013), a obesidade é classificada em: exógena (que engloba fatores externos socioambientais) e endógena (que tem relação aos fatores genéticos ou neuroendócrinos com desequilíbrio hormonal).

Cabe ressaltar ainda que as síndromes genéticas e diferentes transformações endocrinológicas se responsabilizam somente por $1 \%$ dos casos de obesidade endógena, entretanto, os outros $99 \%$ são de causas exógenas, sendo principalmente consequência do consumo descontrolado de alimentos (VILAS BOAS, 2016).

O balanço energético positivo, ou superávit calórico, é o excesso de ingestão de alimentos e diminuição do dispêndio energético diário, que está diretamente relacionado com o ganho de peso (RICCO et al., 2019).

Portanto, o motivo do crescimento de massa gorda corporal resulta em uma falta de equilíbrio energético entre as calorias consumidas e as calorias gastas. Este excesso proporciona um padrão nutricional com exagerado consumo calórico, com mínimo consumo energético no dia a dia ou estas duas ocasiões em conjunto (DUNCAN et al., 2012). Para se obter um déficit calórico é necessária a diminuição da ingestão de alimentos com valores elevados de caloria e/ou inserção da AF (RICCO et al., 2019).

Podemos relacionar a obesidade infantil em dois fatores, sendo externos: inatividade física, acesso e publicidade de alimentos industrializados; e fatores internos: hereditariedade, interrupção precoce do aleitamento materno exclusivo, prematuridade e uso de leite de vaca antes de um ano de idade (CARDOSO et al., 2019; WEFFORT e LAMOUNIER, 2017). 
Pesquisas focadas nos alimentos que mais são consumidos pelos brasileiros, evidenciam que os industrializados somam os 10 alimentos que mais são ingeridos pelos adolescentes, sendo pouco encontrados alimentos naturais. Esses hábitos alimentares dos dias atuais, fazem com que haja uma alimentação rica em açúcares, gorduras e alimentos ultra processados, pobre em fibras, legumes e frutas. O referido assim, padrão de alimentação inadequada influencia diretamente no crescimento dos números de excesso de peso (MENDRICK et al., 2018).

Além de todas as questões mencionadas anteriormente, vários estudos questionam se a composição da microbiota intestinal seria causa ou consequência da obesidade. Têmse atribuído a composição da microbiota intestinal a fatores ambientais para o controle do peso corporal (CARVALHO et al., 2013) e a obesidade estaria relacionada também a modificações da microbiota intestinal (disbiose) (NOGUEIRA-DE-ALMEIDA et al., 2020).

Neste sentido, além da obesidade ser considerada uma doença multifatorial, a microbiota intestinal pode ser um fator crucial no desenvolvimento da obesidade. Sabese que $95 \%$ de todas as bactérias encontradas no intestino humano podem ser classificadas em um grupo de sete famílias bacterianas representativas. Um estudo demonstrou que a obesidade, inflamação e lipídios foram associados a menor abundância de Bacteroidaceae, Porphyromonadaceae e Prevotellaceae e maior abundância de Lactobacillaceae quando comparadas a crianças com peso normal. Foi observada uma relação direta entre a abundância de Lactobacillaceae em crianças com obesidade visceral, enquanto uma associação inversa foi observada entre essa família e os níveis de Lipoproteína de alta densidade (HDL). A análise da microbiota em nível familiar pode ser usada para determinar sua associação com obesidade e marcadores de risco metabólico (AGUILAR et al., 2020).

Outro fator com grande responsabilidade na crescente obesidade infantil são as mídias de alimentos de fast-foods. Pode-se atribuir a ela uma parcela da responsabilidade de ocorrência de problemas causados pela má alimentação da população infantil. Encorajando esses indivíduos ao consumo desses alimentos, resultando em um baixo consumo de alimentos saudáveis (REIS, VASCONCELOS e BARROS, 2011).

Neste sentido, a resolução 24/2010 da Agência Nacional de Vigilância Sanitária ANVISA (BRASIL, 2010), possui a relevante intenção de impossibilitar ou diminuir a elevação de ocorrência de DCNT, especialmente no caso de crianças e adolescentes, 
indivíduos abalizados como de maior vulnerabilidade às mensagens publicitárias. Dessa maneira, toda propaganda dessa espécie precisaria ser acompanhada de alertas referentes aos perigos do consumo em elevadas quantidades desses nutrientes, através da apresentação de mensagens que se adequam às determinações da lei (REIS, VASCONCELOS e BARROS, 2011).

Além das propagandas dos alimentos industrializados, é preciso salientar que a alimentação infantil sofre influência direta dos hábitos alimentares familiares. Sendo assim, é nítido que os hábitos alimentares das crianças são embasados nos pilares das opções inadequadas de seus pais. Neste sentido, a nutrição precisa ser trabalhada em conjunto com os familiares, para que todos passem a adquirir melhores hábitos alimentares (INGE et al., 2013).

Segundo Wada (2019), estudos realizados com crianças de seis e sete anos demonstraram o uso prolongado de aparelhos eletrônicos, sendo que esse uso deve ser administrado e não deve ultrapassar duas horas por dia para crianças nesta fase (ANS, 2017), pois está associado ao maior risco de sobrepeso (WADA et al., 2019).

A realidade escolar influencia consideravelmente no hábito alimentar da criança, não apenas por conta do extenso tempo de permanência, mas ainda por conta do local de convívio e troca de informações (AZEVEDO e BRITO, 2012).

As estratégias para a prevenção da obesidade infanto-juvenil devem se basear no desenvolvimento de ações educativas de promoção da alimentação saudável desde o prénatal; promoção do aleitamento materno; introdução adequada de alimentação complementar; estímulo ao conhecimento sobre a importância da AF e práticas corporais no desenvolvimento da criança e do adolescente; promoção de AF lúdicas e recreativas; observação do comportamento sedentário; promoção adequada de horas de sono; controle do tempo de tela e identificação dos pacientes de risco (ANS, 2017).

\section{Consequências da obesidade Infantil}

De fato, o sobrepeso e a obesidade na infância trazem consequências de natureza física e psicológica. Apresentam uma maior probabilidade de permanecerem obesos até a idade adulta e mais propensos a desenvolver DCNT. Porém algumas doenças relacionadas à obesidade já estão presentes na infância e adolescência como a HAS, dislipidemias, sofrimento mental, apartamento sociais, dores articulares, entre outras. Crianças e adolescentes com obesidade sofrem com consequências a curto, médio e longo prazo (GINANI, BORTOLINI e FELDENHEIMER, 2021). 
Além das condições comórbidas e médicas que envolvem a obesidade infantil, ela tem sido apresentada como uma das condições mais estigmatizantes e menos socialmente aceitáveis na infância. Crianças com sobrepeso e obesas são frequentemente provocadas e/ou intimidadas por seu peso. Eles também enfrentam inúmeras outras dificuldades, incluindo estereótipos negativos, discriminação e marginalização social (MACHADO, 2014).

A literatura descreve que a discriminação contra indivíduos obesos foi encontrada em crianças a partir dos 2 anos de idade. Em seu contexto social são frequentemente excluídas de atividades competitivas, que necessitam da AF. Geralmente, é difícil para crianças com sobrepeso participarem de AF, pois tendem a ser mais lentas que seus pares, pois enfrentar falta de ar, dificuldade de locomover-se, são mais lentas (SAHOO et al., 2015). Dessa forma, bullying, baixa autoestima, redução na frequência escolar, contribuem futuramente para a empregabilidade na vida adulta.

Crianças obesas, em relação às eutróficas, caracterizam-se por apresentarem apetite aumentado, mais agressividade, interesse pela sexualidade, ansiedade, tendência à depressão, piores relações interpessoais, acentuado desequilíbrio afetivo, débil tolerância às frustrações e grau de autoestima muito inferior ao das crianças não obesas (LAMOUNIER, CHAGAS e VIEIRA, 2005). O excesso de peso pode coexistir ainda com outras formas de má nutrição, incluindo carência de micronutrientes e déficit de estatura para a idade (CARDOSO et al., 2019). Além de apresentarem pior qualidade de vida quando comparados com indivíduos saudáveis da mesma idade.

Existem inúmeras informações relacionadas a obesidade na infância e sua continuação durante a adolescência e a fase adulta, assim como a ocorrência de diferentes comorbidades no decorrer da vida. As doenças cardiovasculares, DM2 e câncer, acabam representando $63 \%$ de todas as mortes em todo o mundo. Sendo que o sobrepeso e obesidade que acometem crianças são os mais relevantes motivos que resultam para a obesidade na idade adulta e surgimento de DCNT (INGE et al., 2013).

As doenças cardiovasculares só se manifestam décadas mais tarde, mas os fatores de risco surgem na infância e estão diretamente relacionados ao excesso de peso e à distribuição de gordura, assim como nos adultos (ABESO, 2016). As complicações cardiovasculares mais comuns são a HAS e a hipertrofia cardíaca (VILAS BOAS, 2016).

\section{Atividade física}


Devido aos eletrônicos e ao melhor acesso à escola, as oportunidades dos indivíduos ser fisicamente ativo diminuíram nos últimos anos. No passado, grande parte das crianças frequentavam a escola caminhando ou de bicicleta. Atualmente este cenário mudou devido à distância, à falta de rota segura para caminhada e ao transporte escolar (SAHOO et al, 2015).

A prática de $\mathrm{AF}$ no enfrentamento da obesidade durante a infância e a adolescência, pode contribuir de três maneiras: I) AF nessa fase auxilia no equilíbrio do balanço energético; II) jovens ativos tendem a se tornar adultos ativos; III) jovens ativos possuem menor probabilidade de desenvolver obesidade e suas comorbidades na fase adulta, enquanto os inativos têm mais de $90 \%$ de chance de se tornarem adultos sedentários (HEARNSHAW e MATYKA, 2010).

Os benefícios da AF vão além do controle da obesidade. Ela se constitui num componente importante da prevenção e tratamento da doença coronariana, da HAS, das doenças musculoesqueléticas, das doenças respiratórias e da depressão (YUMUK et al., 2015). Kelley et al. (2011), em uma análise na literatura, buscaram determinar os efeitos do exercício físico, associado a uma dieta, sobre as concentrações de lipídios e lipoproteínas, constatando que a combinação de exercício e dieta são eficazes para melhora dos níveis de colesterol total, LDL e triglicerídeos, mas não observaram melhoras nos níveis de HDL.

Tem sido demonstrado que a prática regular de AF é também eficiente na redução e controle da pressão arterial (PA) em crianças. Estudos mostram que exercícios aeróbios, que devem ser complementados pelos resistidos, reduzem a PA e existe uma associação positiva entre aumento do IMC e PA em crianças (HELENO et al., 2017).

Outro estudo demonstrou que a redução da HAS decorre de um efeito direto do exercício físico sobre os níveis pressóricos, independentemente das alterações do peso corporal ou percentual de gordura (FARPOUR-LAMBERT, et al., 2009).

Rondom e Brum (2003), em pesquisa avaliando o exercício físico como um meio para o tratamento da HAS, apontam que o exercício regular é capaz de reduzir ou mesmo acabar com a necessidade de uso de medicamento, contribui para evitar os efeitos adversos do tratamento farmacológico e reduz o custo do tratamento para o paciente e para as instituições de saúde.

Os efeitos biológicos relacionados a altos níveis de AF em crianças são baixa pressão sanguínea, níveis séricos de lipídios e lipoproteínas mais favoráveis, mais 
sensibilidade à insulina e menos adiposidade, como também é considerada importante fator para atingir e manter adequada resistência óssea, contribuindo para o desenvolvimento esquelético normal (ANDERSEN, 2009). Uma vez que a obesidade é considerada uma doença inflamatória e o exercício físico modula de forma direta tais processos, é essencial a implementação de programas de exercícios físicos na melhora da resposta inflamatória de obesos (PRADO et al., 2009).

\section{CONSIDERAÇÕES}

Frente ao exposto neste trabalho, acreditamos ser necessárias investigações em saúde e nutrição que valorizem abordagens metodológicas que sejam introduzidas na perspectiva da obesidade enquanto uma enfermidade multifatorial, como normalmente se apresenta na literatura. Neste sentido, embora algumas literaturas já tenham realizado estudo com abordagem mais abrangente e no plural sobre a obesidade infantil relacionando sobre fatores internos e externos.

Já que a prevenção de doenças cardiovasculares e metabólicas é comumente baseada em mudanças nos parâmetros antropométricos, composição corporal ou níveis de lipídios e glicose, essas medidas de resultado podem ser insuficientes. A nosso ver, é a partir da construção de estudos dessa natureza que será possível promover uma instrumentalização mais eficiente dos profissionais da equipe multidisciplinar para o tratamento do sobrepeso e obesidade infantil no nível individual e familiar, no qual as abordagens multidisciplinares e intersetoriais assumiram destaque.

Ações no âmbito coletivo devem haver mais políticas públicas que promovam a saúde, o bem-estar e a qualidade de vida onde atende mais a sociedade civil, sendo um caminho promissor na prevenção e tratamento da obesidade, por meio da responsabilização e do autocuidado, permitindo que a comunidade participe do processo de promoção da saúde. A obesidade em idade pediátrica precisa e deve ser considerada como um evento prioritário de controle, visando a prevenção de doenças que se desenvolvem associadas a esse problema.

A partir da revisão da literatura, fica evidente que diversos fatores têm contribuído para o aumento significativo da obesidade, sendo observada e descrita desde o século passado como um agravo à saúde da população de forma geral.

Dessa forma, a participação em programas de AF deve de acordo com a faixa etária e orientação nutricional devem ser incentivada na criança para reduzir problemas relacionados à doença. 
Pesquisas futuras devem investigar o volume, a intensidade e a duração da AF necessária para melhorar as funções endoteliais, cardiovasculares e metabólicas neste público infantil.

\section{REFERÊNCIAS}

ABESO - Associação Brasileira para o Estudo da Obesidade e da Síndrome Metabólica. Diretrizes Brasileiras de Obesidade - Associação Brasileira para estudo da obesidade e síndrome metabólica. 4.ed. - Itapevi, SP, 2016.

ABESO - Associação Brasileira para o Estudo da Obesidade e da Síndrome Metabólica. Mapa da Obesidade. São Paulo: ABESO, 2019. Disponível em: <www.abeso.org.br>. Acesso em 21 de janeiro de 2022.

ANDERSEN, L.B. Physical activity in adolescents. J Pediatr, v. 85, n. 4, p. 281-283, 2009.

ANS - Agência Nacional de Saúde Suplementar. Manual de diretrizes para o enfrentamento da obesidade na saúde suplementar brasileira. Rio de Janeiro, 2017.

AGUILAR, T. et al. Gut Bacterial Families Are Associated with Body Composition and Metabolic Risk Markers in School-Aged Children in Rural Mexico. Childhood Obesity, v. 16, n. 5, 2020.

AZEVEDO, F.R. BRITO, B.C. Influência das variáveis nutricionais e da obesidade sobre a saúde e o metabolismo. Revista da Associação Médica Brasileira, v. 58, n. 6, p. 714 $-723,2012$.

BRASIL. Ministério da Saúde. Agência Nacional De Vigilância Sanitária. Diretoria Colegiada. RESOLUÇÃO-RDC No 24, de 15 DE JUNHO de 2010. Dispõe sobre a oferta, propaganda, publicidade, informação e outras práticas correlatas, 2010.

CARDOSO, A. et al. Panorama da obesidade em crianças e adolescentes. Instituto Desiderata, v. 1, n. 1, 2019.

CARVALHO, E.A.A. et al. Obesidade: aspectos epidemiológicos e prevenção. Revista Médica de Minas Gerais, v. 23, n. 1, p. 74 - 82, 2013.

CAMPAGNOLO, P.D.B. et al. Prevalence of overweight and associated factors in southern Brazilian adolescents. Public Health, v. 122, n. 5, p. 509-515, 2008.

CONDE, W.L. MONTEIRO, C.A. Body mass index cutoff points for evaluation of nutritional status in Brazilian children and adolescents. Jornal de pediatria, v. 82, p. 266-272, 2006.

CRESCENTE, C.L. et al. Prevalência de obesidade infantil: há motivo de preocupação? Saúde e Pesquisa, v. 14, n. 3, p. 1-11, 2021. Disponível em: < https://177.129.73.3/index.php/saudpesq/article/view/8606>. Acesso em: $05 \mathrm{dez} . \mathrm{de}$ 2021. 
DUNCAN, B.B. et al. Doenças crônicas não transmissíveis no Brasil: Prioridade para enfrentamento e investigação. Revista de Saúde Pública, v. 46, n.1, p. 126-134, 2012.

FARPOUR-LAMBERT, N.J. et al. Physical activity reduces systemic blood pressure and improves early markers of atherosclerosis in pre-pubertal obese children. J Am Coll Cardiol., v. 54, n. 25, p. 2396-406, 2009.

GINANI, J. BORTOLINI, G. FELDENHEIMER, A.C. Principais Questões sobre Sobrepeso e Obesidade na Infância. [S. 1.], 12 fev. 2021. Disponível em: $<$ https://portaldeboaspraticas.iff.fiocruz.br/atencao-crianca/principais-questoes-sobresobrepeso-e-obesidade-na-infancia/>. Acesso em: 17 dez. 2021.

GURNANI, M. BIRKEN, C. Hamilton, J. Childhood Obesity. Pediatric Clinics of North America, 2015, 62(4), 821-840. doi:10.1016/j.pcl.2015.04.001. Disponível em: <https://pubmed.ncbi.nlm.nih.gov/26210619/>. Acesso em: 14 dez. de 2021.

HEARNSHAW, C. MATYKA, K. Managing childhood obesity: when lifestyle change is not enough. Diabetes Obes. Metab., v. 12, n. 11, p. 947-957, 2010.

HELENO, P. et al. Systemic arterial hypertension, blood pressure levels and associated factors in schoolchildren. Rev. Assoc. Med. Bras., v. 63, n.10, 2017.

INGE, T.H. et al. The effect of obesity in adolescence on adult health status. Pediatrics, v. 132, n. 6, p. $1098-104,2013$.

JARDIM, J.B. SOUZA, L.S. Obesidade infantil no Brasil: uma revisão integrativa. Disponível em: 〈https://jmphc.com.br/jmphc/article/view/275/419>. Acesso em $10 \mathrm{dez}$. de 2021.

KELLEY, G.A. et al. Efficacy of aerobic exercise and a prudent diet for improving selected lipids and lipoproteins in adults: a meta-analysis of randomized controlled trials. BMC Medicine, v. 9, n. 74, p. 1-15, 2011.

KUMAR S, KAUFMANN T. Childhood obesity. Panminerva Med. 2018 Dec;60(4):200-212. doi: 10.23736/S0031-0808.18.03557-7. Epub 2018 Oct 5. PMID: 30303357. Disponível em: <https://pubmed.ncbi.nlm.nih.gov/30303357/>. Acesso em 14 dez. de 2021.

LAMOUNIER, J.A. CHAGAS, A.J. VIEIRA, E.C. Obesidade. In: Leão E, Correa E, Mota JAC, Viana BV. Pediatria ambulatorial. Belo Horizonte: Coopmed, 2005; p. 1034.

LAMOUNIER, J. A. Nutrição infantil, atividade física e a pandemia pelo Coronavírus. Revista Residência Pediátrica - Ponto de Vista. Novembro. 2021

MACHADO, R. P. Obesidade infantil: como a educação física pode contribuir para a sua prevenção no ambiente escolar. 2014. Disponível em: <https://www.lume.ufrgs.br/handle/10183/116053>. Acesso em: 19 jan. de 2022.

MENDRICK, D.L. et al. Metabolic Syndrome and Associated Diseases: From the Bench to the Clinic. Toxicol Sci., v. 162, n. 1, p. 36-42, 2018.

MENEZES, C.A. VASCONCELOS, R.S. Distanciamento social, risco cardiometabólico e alteração psicossocial em crianças obesas durante a pandemia do COVID-19. Saúde 
Coletiva (Barueri), v. 11, n. 65, p. 5870-5881, 2021. Disponível em: $<$ http://www.revistas.mpmcomunicacao.com.br/index.php/saudecoletiva/article/vie w/1575/1803>. Acesso em 05 dez. de 2021.

NCD Risk Factor Collaboration. Worldwide trends in body-mass index, underweight, overweight, and obesity from 1975 to 2016: a pooled analysis of 2416 population-based measurement studies in 128.9 million children, adolescents, and adults. The Lancet, v. 390, n. 10113, p. 2627-2642, 2017.

NOGUEIRA-DE-ALMEIDA, C.A. et al. COVID-19 and obesity in childhood and adolescence: A clinical review. J Pediatr., v. 96, n. 5, 2020.

OLIVEIRA, E.N. et al. Benefícios da atividade física para saúde mental. Saúde Coletiva, v. 8, n. 50, p. 126-130, 2011.

PAES, S.T. MARINS, J.C.B. ANDREAZZI, A.E. Efeitos metabólicos do exercício físico na obesidade infantil: uma visão atual. Revista Paulista de Pediatria, v. 33, p. 122-129, 2015.

Disponível

em: <https://www.scielo.br/j/rpp/a/KFwvSFP9hsLknRMcZ77CzYB/abstract/?lang=pt>. Acesso em: 19 jan. de 2020.

PEREIRA-LANCHA, L.O.; CAMPOS-FERRAZ, P.L.; LANCHA, A.H.Jr. Obesity: considerations about etiology, metabolism, and the use of experimental models. Diabetes Metab Syndr Obes., v. 5, p. 75-87, 2012.

PRADO, W.L. et al. Obesidade e adipocinas inflamatórias: implicações práticas para a prescrição de exercícios. Rev Bras Med Esporte, v. 15, n. 5, 2009.

REIS, C.E.G. VASCONCELOS, I.A.L. BARROS, J.F.N. Políticas públicas de nutrição para o controle da obesidade infantil. Revista Paulista de Pediatria, v. 29, n. 4, p. 62533, 2011.

RICCO, R.C. et al. Atividade física visando emagrecimento. In: WEFFORT VRS (org.). Manual de Orientação: Obesidade na Infância e Adolescência. Sociedade Brasileira de Pediatria. Departamento Científico de Nutrologia. $3^{a}$. Ed. - São Paulo: SBP. 2019; p.132-144.

RONDOM, M.U.P. BRUM, P.C. Exercício físico como tratamento Exercício físico como tratamento não-farmacológico da hipertensão arterial. Rev Bras Hipertens., v. 10, p. 134-139, 2003.

SBC - SOCIEDADE BRASILEIRA DE CARDIOLOGIA. O que acontece com nossas crianças e jovens obesos?. 2020. Disponível em: <https://www.portal.cardiol.br/post/oque-acontece-com-nossas-crian\%C3\%A7as-e-jovens-obesos $>$. Acesso em: $16 \mathrm{dez}$. de 2021.

SBP - SOCIEDADE BRASILEIRA DE PEDIATRIA. Departamento Científico de Nutrologia. Obesidade na infância e adolescência: manual de orientação. 2. ed. rev. ampl. São Paulo: SBP, 2012; 142 p.

SAHOO, K. et al. Obesidade infantil: causas e consequências. Journal of Family Medicine and Primary Care , v. 4, n. 2, pág. 187, 2015. Disponível em: < https://www.ncbi.nlm.nih.gov/pmc/articles/PMC4408699/>. Acesso em 16 out. de 2021. 
VIGITEL Brasil 2019: Vigilância de fatores de risco e proteção para doenças crônicas por inquérito telefônico. Brasília: Ministério da Saúde, 2020. Disponível em: <http://bvsms.saude.gov.br/bvs/publicacoes/vigitel_brasil_2019_vigilancia_fatores_risc o.pdfㄱ. Acesso em 21 de janeiro de 2022.

VILAS BOAS, A.B.B. Como a educação física e exercício físico podem auxiliar no combate contra a obesidade em crianças e jovens. Trabalho de Conclusão de Curso apresentado como requisito parcial para a obtenção do título de Licenciado em Educação Física, 2016.2 Disponível em: $<$ https://www.iessa.edu.br/revista/index.php/tcc/article/download/54/3>. Acesso em: dezembro de 2021.

WADA, K. et al. Associations of Cell Phone Use and Screen Viewing with Overweight in Children. Childhood Obesity, v. 15, n. 7, 2019.

WEFFORT, V.R.S. LAMOUNIER, J.A. (Coord.). Nutrição em pediatria: da neonatologia a adolescência. 2. ed. Barueri, SP: Manole, 2017.

YUMUK, V. et al. European Guidelines for Obesity Management in Adults. Obes Facts, v. 8, n. 6, p. 402-24, 2015. 